\title{
Les différents types de mutations d'un gène et leurs conséquences
}

Mutations ponctuelles

Une mutation ponctuelle est le changement d'une base par une autre. Ses conséquences peuvent être multiples.

$\mathbf{1}, 2$ et 3 . Une mutation des régions de contrôle de l'expression du gène, notamment des boites CAAT et ATA du promoteur, peut modifier la transcription et aboutir à une diminution de la quantité du messager, donc de la protéine correspondante.

4. Une mutation intéressant le codon d'initiation de la traduction (ATG) ou le pentanucléotide le précédant (se rapprochant du consensus $\left.C_{G}^{A} C C\right)$ peut diminuer la traductibilité du messager, donc la concentration de la protéine pour laquelle il code.

5. Une mutation d'une base dans un exon peut être muette si elle ne change pas le sens du codon, aboutir à une protéine altérée si elle change le sens du codon (" mutation faux-sens "), ou à une absence de protéine si elle fait apparaitre un codon stop très proche du début de la traduction. Une semblable " mutation non sens " située près de la fin normale de la protéine pourrait aboutir à la synthèse d'un produit raccourci. Enfin, la conséquence d'une mutation ponctuelle exonique peut être l'apparition d'un site alternatif d'épissage (voir médecine/sciences, prochain Lexique : mécanismes des anomalies d'épissage).

6. Une modification des bases GT situées au début de l'intron supprime ce site d'épissage (voir médecine/sciences, prochain Lexique). 7,8. Une mutation intronique est le plus souvent muette; elle peut aussi faire apparaitre un site alternatif d'épissage (voir prochain Lexique). 9. Une mutation du doublet AG situé à la fin des introns abolit ce site d'épissage (voir $\mathrm{m} / \mathrm{s}$, prochain Lexique).

I0. Une mutation du codon stop supprime l'arrêt de la traduction à ce niveau. L'ARN est donc traduit jusqu'à ce qu'un nouveau codon stop soit rencontré, la conséquence étant la production d'une protéine allongée.

II. Une mutation du signal de polyadénylation AATAA peut diminuer la proportion des transcrits qui sont correctement clivés, puis polyadénylés. Les ARN ayant subi une maturation anormale sont instables, et la quantité du messager fonctionnel sera diminuée.

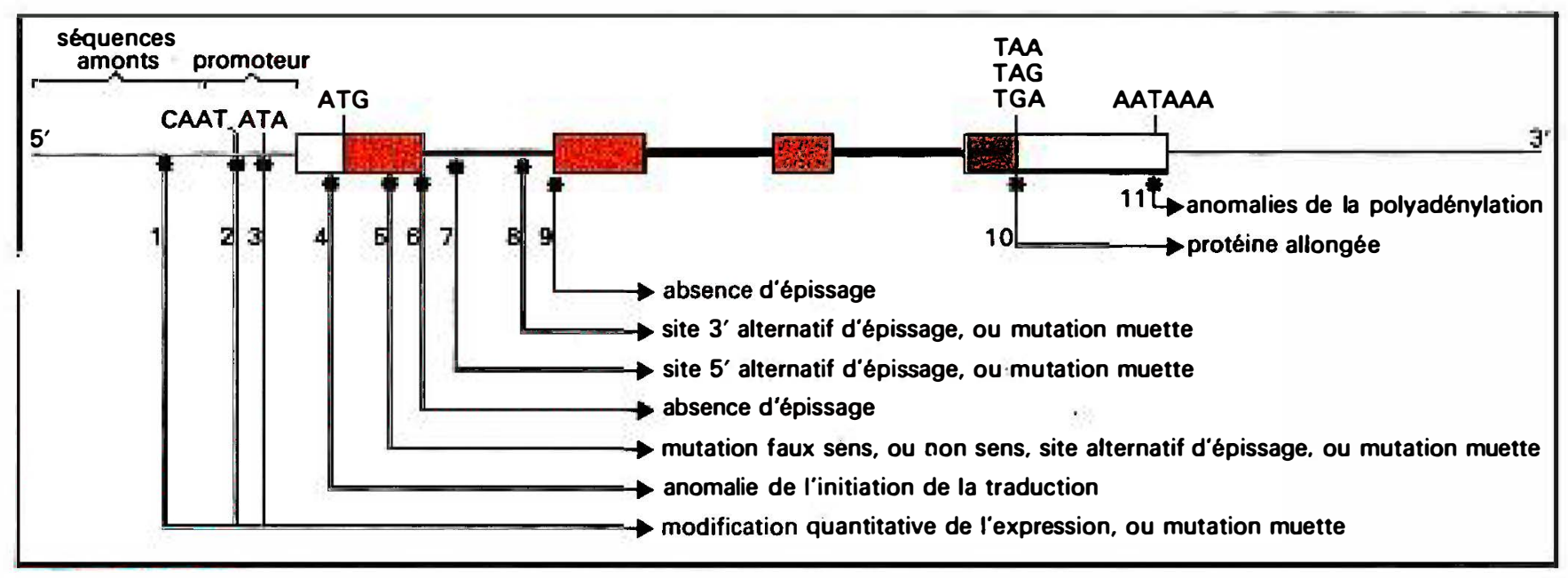

Figure 1. Conséquences possibles des mutations ponctuelles d'un gène et de ses régions régulatrices. Les traits fins représentent les régions intergéniques, les traits épais les introns, les rectangles blancs la partie non codante des exons et les rectangles rouges la partie codante des exons. Les "boîtes "CAAT et ATA du promoteur sont représentées, ainsi que le codon d'initiation de la traduction ATG, les codons stop TAA, TAG ou TGA, et le signal de polyadénylation AATAAA. 


\section{Délétions et insertions}

Les délétions de tout un gène ou d'un fragment important d'ADN sont souvent dues à un crossing-over non équationnel entre deux régions non alléliques partiellement ou totalement homologues, selon le mécanisme illustré dans le Lexique de médecine/sciences $n^{\circ} 4$, vol. I, p. 215, fig. 2 . Elles aboutissent à une perte de la fonction du gène.

Des microdélétions de quelques bases peuvent, si elles emportent un multiple de 3 bases dans un exon, aboutir à la synthèse d'une protéine anormale, à laquelle manqueront quelques aminoacides. Si la délétion supprime un non-multiple de 3 bases, elle créera un décalage de phase de lecture et, en aval de la mutation, le sens des codons sera entièrement modifié; ils seront traduits en une protéine n'ayant stric- tement rien à voir avec celle normalement codée par le gène (voir médecine/sciences, prochain Lexique).

Les insertions sont des phénomènes rares qui peuvent être dus à l'intégration dans un gène d'un rétrovirus, ou d'un élément transposable. Les éléments transposables, surtout connus chez les procaryotes, les plantes et les drosophiles, sont des segments d'ADN possédant à leurs deux extrémités des séquences répétées (homologues des LTR des rétrovirus), qui ont la propriété d'être excisés du génome et de se réintégrer en d'autres sites. Certaines séquences répétitives des mammifères pourraient avoir cette propriété.

Une insertion peut également être secondaire à une duplication partielle d'un gène. Les conséquences sont parallèles à celles d'une délé- tion et, pour de très pétites insertions, varient selon que le fragment inséré contient un multiple de trois bases (entraînant la synthèse d'une protéine altérée) ou un nonmultiple de trois bases (produisant un décalage de phase de lecture).

Fréquences relatives des différents types de mutation

Elles varient évidemment selon les gènes mutés. Très approximativement, les délétions représentent de 5 à $15 \%$ des mutations connues chez l'homme.

Parmi les mutations ponctuelles, certaines sont très rares (mutations des zones de contrôle). Les plus habituelles sont sûrement les mutations faux-sens et les anomalies d'épissage.

A. $\mathbf{K}$. 